\title{
Interview with Richard P. Stanley
}

Toufik Mansour

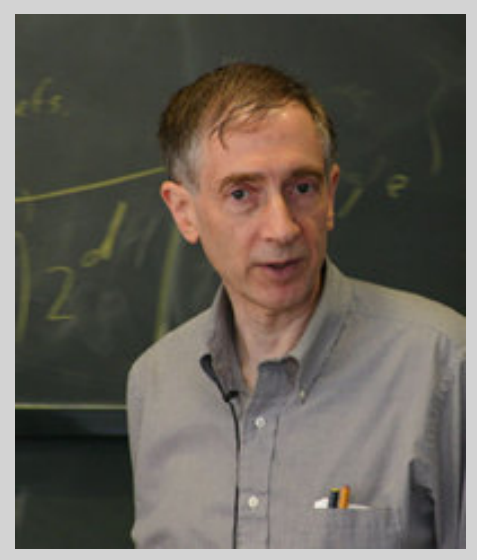

Richard P. Stanley received a B.S. in mathematics from Caltech in 1966 and a Ph.D. in mathematics from Harvard in 1971 under the supervision of Gian-Carlo Rota. Professor Stanley's research concerns problems in algebraic combinatorics, and his distinctions include the 1975 SIAM Pólya Prize in Applied Combinatorics, membership in the National Academy of Sciences since 1995, the 2001 Leroy P. Steele Prize for mathematical exposition, the 2003 Rolf Schock Prize in Mathematics, a plenary lecture at the International Congress of Mathematicians in 2006, and election as a fellow of the American Mathematical Society in 2012.

Mansour: Professor Stanley, first of all we would like to thank you for accepting this interview. Would you tell us broadly what combinatorics is?

Stanley: Not so easy! No definition will be entirely satisfactory, but I think of combinatorics as the study of discrete objects with only simple additional structure. A symmetric binary relation $R$, not allowing $x R x$ but with no further conditions, is quite simple, and this defines the combinatorial concept of simple graphs. On the other hand, a group has too much "additional structure" to be considered a pure combinatorial object. If you combine in a suitable sense simple and more complicated structures, then you get subjects like algebraic, geometric, and topological combinatorics.

Mansour: What do you think about the development of the relations between combinatorics and the rest of mathematics?

Stanley: There has been fantastic development since I started doing combinatorics in the 1960's. Algebraic combinatorics by definition involves the relationship between combinatorics and algebra. It is now a major subarea of combinatorics, as attested by the Mathematics Subject Classification Sys- tem (MSC2020) of Math Reviews and Zentralblatt. In this scheme combinatorics is divided into five areas, one of which is algebraic combinatorics. Almost every area (maybe even every area) of mathematics that involves a lot of algebra is connected with algebraic combinatorics. These areas include, for instance, algebraic geometry, representation theory, and algebraic topology. Moreover, some areas that traditionally were not very algebraic, such as convex polytopes, have in recent years become deeply entwined with algebraic combinatorics.

Of course areas of combinatorics beside algebraic combinatorics also have a deep relationship with other parts of mathematics. I feel less qualified to discuss these, but one example that really stands out is the use of Szemerédi's theorem by Green and Tao to show that there exist arbitrarily long arithmetic progressions of primes.

All these connections are great examples of the unity of mathematics and should provide strong motivation for beginning researchers to learn as much mathematics as possible.

Mansour: What have been some of the main goals of your research?

Stanley: I don't have any overall goals, but

The authors: Released under the CC BY-ND license (International 4.0), Published: October 19, 2020

Toufik Mansour is a professor of mathematics at the University of Haifa, Israel. His email address is tmansour@univ.haifa.ac.il. 
when I get interested in a problem I want to do more than just solve it; I want to really understand what's going on and to be able to use the solution to proceed further.

As an example, after Paul Edelman showed me that the number of reduced decompositions of the longest element $w_{0}$ of the symmetric group $\mathfrak{S}_{n}$ factors into small factors for some small values of $n$, I felt that something really new and fundamental must be involved. I didn't want just to find (and prove) the answer. A real understanding would hopefully say something about the number of reduced decompositions of any element of $\mathfrak{S}_{n}$, as well as elements of other Coxeter groups. Perhaps it would tie in with other objects related to the symmetric group, such as symmetric functions and representation theory. I did succeed in solving the problem and in achieving some understanding by tying it in with symmetric functions. However, I wasn't entirely satisfied with the solution because the proof that a certain quasisymmetric function was actually a symmetric function was very $a d$ hoc and unenlightening. Afterwards many other persons made further contributions by connecting the problem with representation theory, algebraic geometry, nil-Coxeter algebras, a version of RSK, etc. Now we have an excellent understanding of reduced decompositions. For instance, it is clear from this general understanding that for the Coxeter group of type $D_{n}$, we need to weight the reduced decompositions by certain powers of two.

Another example is the Anand-DumirGupta conjecture, namely, the number $H_{n}(r)$ of $n \times n$ matrices of nonnegative integers for which every row and column sum equals $r$ is a polynomial in $r$ of degree $(n-1)^{2}$ (with some additional properties). Again, when I first saw this conjecture I had a strong feeling that there was some deep underlying general principle, and my goal was to find a solution at this level. I did succeed in finding a good reason for the polynomiality, namely, $H_{n}(r)$ is the Hilbert polynomial of a certain graded algebra. Actually, this argument only showed that $H_{n}(r)$ is a polynomial for $r$ sufficiently large. I found an ad hoc argument that polynomiality held for all $r$, as well as for the additional proper-

\footnotetext{
*https://bookstore.ams.org/cworks-25/1

${ }^{\dagger}$ https://link.springer.com/article/10.1007/s00026-014-0238-5
}

ties conjectured by Anand, Dumir, and Gupta. Later further understanding was achieved by connecting $H_{n}(r)$ with Ehrhart theory; in fact, $H_{n}(r)$ is the Ehrhart polynomial of a certain polytope. Moreover, the theory of toric varieties provides a link between the commutative algebra and the polytope approaches. This is the kind of general understanding that I strive for.

Mansour: What were your early experiences with mathematics?

Stanley: This question and the next three are dealt with in my two papers:

The early years, Selected Works of Richard P. Stanley (P. Hersh, T. Lam, P. Pylyavskyy, and V. Reiner, eds.), American Mathematical Society, Providence, RI, 2017, pp. 1-2.*

How the Upper Bound Conjecture was proved, Annals of Combinatorics 18 (2014), 533-539 ; reprinted in Selected Works of Richard P. Stanley (P. Hersh, T. Lam, P. Pylyavskyy, and V. Reiner, eds.), American Mathematical Society, Providence, RI, 2017, pp. 3-10.*

Mansour: Were there specific problems that made you first interested in combinatorics?

Stanley: As I explain in the second paper just mentioned, the first combinatorial problem to attract my interest, even though at that time I had no interest in working in combinatorics, was to explain the similarity between two generating functions: $1 /\left(2-e^{x}\right)$ is the exponential generating function for ordered set partitions of an $n$-element set, and $1 /(2-\zeta(s))$ (where $\zeta(s)$ is the Riemann zeta function) is the Dirichlet series generating function for the number of ordered factorizations of a positive integer. Perhaps the next such problem was the enumeration of solid (3-dimensional) partitions, generalizing MacMahon's famous enumeration of plane partitions. I never made significant progress (and most likely the problem is intractable), but it did lead me to the theory of $P$-partitions, the subject of my Ph.D. thesis.

Mansour: We would like to ask you about your formative years. What was the first reason you become interested in mathematics and specifically combinatorics? Did that happen under the influence of your family, or some other people? 
Stanley: See the two papers referenced above. Mansour: How did you end up at Harvard for your Ph.D. and how did you come to work with your adviser, Gian-Carlo Rota?

Stanley: ditto

Mansour: What was the problem you worked on in your thesis?

Stanley: This was the theory of $(P, \omega)$ partitions, where $P$ is a $p$-element poset and $\omega: P \rightarrow\{1, \ldots, p\}$ is a bijection. A $(P, \omega)$ partition of $n$ is a map $\sigma: P \rightarrow \mathbb{N}=\{0,1, \ldots\}$ satisfying $\sigma(s) \geq \sigma(t)$ if $s \leq t$ in $P$, and $\sigma(s)>\sigma(t)$ if $s<t$ and $\omega(s)>\omega(t)$, such that $\sum_{t \in P} \sigma(t)=n$. You can think of $(P, \omega)$-partitions as interpolating between ordinary integer partitions (when $P$ is a chain) and compositions (when $P$ is an antichain). I was interested in the generating function $F_{P, \omega}(x)=\sum_{n \geq 0} f(n) x^{n}$, where $f(n)$ is the number of $(P, \omega)$ partitions of $n$. (Initially I only considered the case when $\omega$ is natural, i.e., $\omega(s)<\omega(t)$ if $s<t$.) It was easy to show that

$$
F_{P, \omega}(x)=\frac{W_{P, \omega}(x)}{(1-x)\left(1-x^{2}\right) \cdots\left(1-x^{p}\right)},
$$

where $W_{P, \omega}(x)$ is a polynomial with integer coefficients. I conjectured that the coefficients were nonnegative. After a lot of work I realized that the coefficients had a simple combinatorial interpretation in terms of linear extensions of $P$. This was the big breakthrough in developing the theory.

Mansour: How was the mathematics at Harvard at that time?

Stanley: There was hardly any combinatorics. Andrew Gleason had some interest in this area, and Garrett Birkhoff did a lot of work in lattice theory, though mostly with infinite lattices. Edward Bender was a postdoc who taught a graduate course in combinatorics (probably the second combinatorics course ever taught at Harvard, the first being by Alfred Hales in 1965) with Jay Goldman, who was in the Department of Statistics. I went to Harvard intending to work on finite groups with Richard Brauer. Although Brauer was a very nice person and would have been a fine adviser, I didn't like the kind of intricate case-by-case analysis that was becoming predominant in the area. I switched to number theory with John Tate as my adviser. I was learning algebraic number theory when I first met Gian-Carlo Rota at the Massachusetts Institute of Technology (MIT), who ended up as my de facto thesis adviser. (My official Harvard adviser was Tate, who agreed to do so if he didn't have to read my thesis! A more natural choice would have been Birkhoff, but Rota warned me that Birkhoff makes all his students rewrite their theses, sometimes several times.)

A couple of courses that I took at Harvard stand out in my mind. The first was a beginning graduate course on complex variable theory taught by David Mumford, which included a proof of the prime number theorem. The second course was a beginning course on algebraic topology taught by Albrecht Dold, who was visiting for the academic year. The first two-thirds of the course was devoted to homology theory and was excellent. The last one-third was on homotopy theory. Dold decided to base the presentation on a pet topic of his, pro-representable functors. As a result, at the end of the course I had no idea how to compute the fundamental group of a torus! Instead of a final exam, we had to write a paper on some aspect of algebraic topology. By then I had switched my main interest to combinatorics, so my paper was on the homology of finite topological spaces.

Algebraic geometry was a major area of the Harvard Mathematics Department when I was there. I audited the last course taught by Oscar Zariski, on algebraic curves. This turned out to be very lucky for me, because of the Minor Thesis requirement at Harvard. When a graduate student felt ready to write a Minor Thesis, he or she was given a topic based on the courses taken so far. The topic was supposed to be unrelated to the courses, so the student had to learn something entirely new. The Minor Thesis had to be handed in three weeks after it was assigned. Since I had only audited Zariski's course (rather than taking it for credit), it did not appear on my record. Therefore I was given the topic "the RiemannRoch theorem for curves." This turned out to be a central result in Zariski's course, so I didn't have to do much more than transcribe my notes.

The Mathematics Department was located on the second floor of Two Divinity Avenue. There wasn't much space, and graduate students did not have offices. If we needed to have 
office hours for the classes we taught, we went to a house a couple of blocks away. I spent a lot of time in the Common Room involved in such activities as blitz chess, bridge, table shuffleboard, and foosball.

My time at Harvard took place during the Vietnam War. Many of the graduate students in the Mathematics Department were extremely politically active. I got converted from a conservative into a liberal who opposed the war. I was involved in some political activity such as the famous march on Washington in November, 1969, and on another occasion I spent a couple of hours in jail (with a lot of other people) for disturbing a public assembly, before bail was raised. Eventually I was fined $\$ 10$. The most significant political event at Harvard when I was a graduate student was the occupation of University Hall, the main administration building. (See for instance the Wikipedia article "Harvard University and the Vietnam War.") I happened to be out of town attending a math meeting, so I was not involved. (The decision to occupy University Hall was made only on the day of the event.) My two roommates were both expelled from Harvard, but later they were allowed to submit theses and obtain their Ph.D. degrees.

Mansour: Would you say a little bit about your most influential results and why they have been influential?

Stanley: My most influential results are all influential for basically the same reasons: the concepts and techniques turn out to be useful in other contexts, and they suggest extensions and generalizations for which much further progress can be made. These results are: (1) the theory of $P$-partitions, (2) combinatorial reciprocity, (3) applications of commutative algebra (especially face rings) and algebraic geometry (mainly, the hard Lefschetz theorem) to combinatorics, (4) stable Schubert polynomials (also called "Stanley symmetric functions") and their connection with reduced decompositions of permutations, and (5) chromatic symmetric functions. There is some other work that has had some influence, such as supersolvable lattices, order and chain polytopes, D-finite power series, a combinatorial interpretation of Schubert polynomials (with Sara Billey and William Jockusch), and a conjectured formula (proved by Valentin Féray) for the normalized character values of the symmetric group.

Mansour: What would guide you in your research? A general theoretical question or a specific problem?

Stanley: Usually they are specific problems, but sometimes the solutions suggest a more general theory. If I have a new idea, I like to stretch it as far as possible.

Mansour: When you are working on a problem, do you feel that something is true even before you have the proof?

Stanley: Often there is good reason to believe that something is true. This is especially the case for enumerative problems when a conjectured answer is known. If some sequence agrees with another in the OEIS for the first ten terms, then they're likely to agree forever. Mansour: What three results do you consider the most influential in combinatorics during the last thirty years?

Stanley: I have to restrict myself to enumerative and algebraic combinatorics (EAC) since I don't have sufficient knowledge of extremal combinatorics, probabilistic combinatorics, etc. There is one exception I would like to mention: the work of Larry Guth on the polynomial method, in particular, the solution (with Nets Katz) to the Erdös distinct distances problem. Even in EAC there is so much happening that it's almost impossible to pick three most influential results. Some contenders are (1) the theory of cluster algebras by Fomin and Zelevinsky (including its connections with total positivity), (2) the work of Bergeron, Garsia, Haiman, and others on diagonal harmonics and the $n$ ! conjecture (especially Haiman's proof of the $n$ ! and $(n+1)^{n-1}$ conjectures), (3) Postnikov's theory of generalized permutohedra, and (4) the work of June Huh and his collaborators on "combinatorial Hodge theory" and related concepts.

Mansour: What are the top three open questions in your list?

Stanley: Three that I find especially interesting are (1) finding a combinatorial interpretation of Schubert polynomial product coefficients, (2) the "Stanley-Stembridge conjecture" on the e-positivity of the chromatic symmetric function of certain graphs, and (3) showing that Young's lattice is the smallest differential poset. This third problem is of little 
significance for the development of combinatorics, but it is a pet interest of mine. A more serious problem would be proving a plethora of conjectures (called "science fiction") by A. Garsia, F. Bergeron, and others extending the theory of diagonal harmonics.

Mansour: What kind of mathematics would you like to see in the next ten-to-twenty years as the continuation of your work?

Stanley: I have no thoughts on this. I am willing to sit back and see what happens.

Mansour: What would you say about some of the major directions in combinatorics for the next two decades?

Stanley: I can only speak about enumerative and algebraic combinatorics. Most of the low-hanging fruit has already been picked, so these areas will become increasingly sophisticated. The next twenty years look bright because there is so much algebraic geometry, representation theory, algebraic topology, etc. (to say nothing of areas like PDE's), that combinatorialists have not yet seriously considered. There is also quite a bit of relatively recent combinatorial machinery, such as combinatorial Hopf algebras and multivariate stable polynomials, whose full potential remains to be seen.

Mansour: Do you think that there are core or mainstream areas in mathematics? Are some topics more important than others?

Stanley: The mainstream areas evolve over time. A mainstream area is one in which lots of progress is continually being made in many directions. Naturally the strong possibility of continuing this progress is going to attract a lot of beginning researchers, so the subject continues to thrive, at least for a while.

Combinatorics as a whole is certainly a mainstream area. There could be some disagreement about which subareas are mainstream. For instance, I feel that design theory, especially the explicit construction of designs, is not so mainstream. Perhaps not everyone will agree. There has been some fantastic recent work in design theory, namely, Peter Keevash's breakthroughs on the existence of designs, but this does not involve explicit constructions.

Algebraic combinatorics is in great shape and should remain mainstream for quite a while. I am somewhat less sanguine about "pure" enumerative combinatorics, i.e., the explicit enumeration (by formulas, recurrences, generating functions, solutions to differential equations, etc.) of combinatorial objects. I can't think of a major recent development in this area, one that opens up a whole new avenue of research. On the other hand, a topic within enumerative combinatorics that seems ripe for further investigation is developing a theory for showing that a given generating function (in one variable) does not have some desirable property, such as being D-finite or differentially algebraic. There are a number of results in this area, but nothing approaching a general theory. The most significant work in this direction (in my opinion) is the disproof of the Noonan-Zeilberger Conjecture by Scott Garrabrant and Igor Pak.

Mansour: What do you think about the distinction between pure and applied mathematics that some people focus on? Is it meaningful at all in your own case? How do you see the relationship between so-called "pure" and "applied" mathematics?

Stanley: I think that there is a difference between pure and applied mathematics, but like almost everything concerning the real world, the idea is vague. Although there is no precise definition of a baby or a mountain, for instance, these concepts are still useful. The same is true of the distinction between pure and applied math. For me a key component of this difference is intention. If someone is doing math with no intent to apply it outside math, and if it is reasonable for he or she to suppose that there is no obvious application, then that person is doing pure math. If X's research on elliptic curves finds an application 50 years later to cryptography, then X's research is still in pure math. The person who found the application would be doing applied math. I also don't feel that it's useful to judge whether or not pure math is "better" than applied. It's like asking whether chemistry is better than poetry. My own work is entirely in pure math, except for a few early papers written when I worked during the summer at the Caltech Jet Propulsion Laboratory.

Mansour: What advice would you give to young people thinking about pursuing a research career in mathematics?

Stanley: Advice could be given at many lev- 
els, such as the rather mundane questions (though important) of what subject to work in, how to choose a thesis adviser, etc. I will say a few words about generating one's own research questions. Unless you are exceptionally brilliant and can solve a long-standing problem of great interest (consider Yitang Zhang ${ }^{\ddagger}$ coming out of nowhere to make a spectacular breakthrough in number theory), it will be really beneficial to your career to produce your own research problems, the more the better (within reason). Always keep your eyes and ears open to possible interesting problems. If for instance a seminar speaker mentions a problem that you like and is more-or-less in an area about which you are knowledgeable, then don't hesitate to think about it! Don't think, "I never worked on hyperconvex residuated posets, so how could I get anywhere?" Play around with it a little - maybe you will think of something. It might suggest a related question. Do some experiments, gather some data, etc. Doing some computations might suggest a further idea, even if the computations themselves don't seem helpful. Moreover, if you decide to stop working on a problem, do not think that you are giving up. You never know when some random remark at a seminar or in a paper might be the key to further progress. Keep these unsuccessful attempts in the back of your mind, ready to be let out if the door is opened a crack.

I have seen numerous instances of young researchers unwilling to work on a problem until they feel that they have mastered all the theory that is associated with it. I don't work this way. I feel that you should dive into working on problems immediately. For instance, suppose you have some interest in polynomials with only real zeros. At a seminar the speaker gives an incomprehensible proof that the Poincaré polynomials of some class of algebraic varieties have a nice combinatorial description. The speaker says that for all cases that could be computed, the polynomials have only real zeros. If the problem of proving that all the polynomials have only real zeros appeals to you, don't feel that you need to understand how they are connected with algebraic geometry. Get to work immediately using the combinatorial description!
Along the same lines, you should also not be afraid to use results whose proofs you don't understand. If you want to understand the proofs, that can come later. First, solve the problem! If it seems that the proof technique might actually be useful, then you can study it. As an example, the key result which I needed to prove the Upper Bound Conjecture for Spheres was a theorem of Gerald Reisner. It wasn't until years later that I actually understood the proof, and that was because I thought it would be applicable to another problem.

I don't want to create the impression that I am against learning proofs in general. Of course mathematicians should be curious about why results are true. I am just suggesting that to use a result in your own research, you don't need to know its proof.

Mansour: Would you tell us about your interests besides mathematics?

Stanley: I have a lot of "small" interests. There is a famous saying going back to the ancient Greek poet Archilochus, "The fox knows many things, but the hedgehog knows one big thing." When it comes to my nonmathematical interests, I am a fox. I have a superficial knowledge or skill about lots of things, but I am nowhere near proficient in any of them. They include table tennis, juggling, magic, cycling, hiking, playing the piano, opera, consciousness, animals, small children, classical history, science fiction, comic books, writing fiction, reading Chinese, bridge, and chess problems.

Mansour: Before we close this interview with one of the foremost experts in algebraic combinatorics, we would like to ask some more specific mathematical questions. You have met several combinatorial objects throughout your career. Which one is your favourite?

Stanley: If symmetric functions and posets qualify as "objects," then it would be these two. They both have a tremendously rich theory. If something more specific is wanted, then two candidates would be Young's lattice and the weak order on the symmetric group. Both of these lattices are intimately connected to the symmetric group, so one could also say that this group is my favorite combinatorial object. (It is actually a hybrid algebraic/combinatorial

\footnotetext{
${ }^{\ddagger}$ https://www.quantamagazine.org/yitang-zhang-and-the-mystery-of-numbers-20150402/
} 
object.)

Mansour: You are interested in Catalan numbers and even wrote a book on it. Why Catalan numbers and not any other well-known combinatorial integer sequences?

Stanley: I certainly am interested in other combinatorial sequences, in particular, Euler numbers and the numbers $(n+1)^{n-1}$. However, Catalan numbers are the most ubiquitous and have by far the most number of elementary properties (such as over 200 combinatorial interpretations), which make them suitable for a problem-format monograph at the undergraduate and graduate student level.

Mansour: The study of permutation patterns has seen great advances in the last thirty years. Any comments on the research in this direction?

Stanley: I am impressed by the growth of this subject. At the time, say, of Julian West's thesis, who could have predicted that there would be a textbook and annual conferences on permutation patterns? There is even a database of pattern avoidance (maintained by Bridget Tenner) analogous to the Online Encyclopedia of Integer Sequences. I especially like the way that pattern avoidance makes numerous appearances connected with the geometry of flag varieties.

Mansour: What about combinatorial aspects of Smith normal form?

Stanley: Whenever you have a combinatorially defined matrix, say over the integers, whose determinant factors nicely, you can ask about its Smith normal form (SNF). There are many such matrices, so lots of nice problems. However, for the most part the SNF does not have any known combinatorial significance, unlike the determinant. Although I enjoy these SNF problems, they seem to be mostly problems in algebra, not combinatorics. An exception is the connection between the SNF of the Laplacian matrix of a graph $G$ and chip-firing on $G$. There are also some applications of SNF to combinatorial designs. It would be great to have some further combinatorial applications of SNF. Even better would be a combinatorial theory of SNF analogous, say, to the Lindström-Gessel-Viennot theory of determinants.

Mansour: You have some interesting results on parking functions and hyperplane arrangements. How do you see these two directions in the next decade?

Stanley: The theory of hyperplane arrangements is a vast area with deep connections to a host of other subjects. I expect the development of these connections to continue to thrive. On the other hand, parking functions are more specialized. However, they are related to such areas as chip firing and diagonal harmonics. These two subjects should continue to expand, but it's unclear to me how much of a role parking functions and their generalizations will play.

Mansour: Is there a specific problem you have been working on for many years? What progress have you made?

Stanley: Since around 1992 I have been thinking about the "Stanley-Stembridge conjecture." Its original formulation was that for certain subsets $S$ of $\mathfrak{S}_{n}$, the sums $\sum_{w \in S} p_{\rho(w)}$ are $h$-positive (using standard symmetric function notation and terminology). The most notable development of my work on this problem was the concept of chromatic symmetric functions. The conjecture can be reformulated as saying that certain chromatic symmetric functions are $e$-positive. However, I have not made any significant contribution to the conjecture itself.

I must confess that from time to time I think about the Riemann hypothesis. I have a crazy idea that almost certainly is worthless, but the payoff is so large that I continue to think about it.

Mansour: Your two-volume book on enumerative combinatorics has been a must-read for many young aspiring combinatorialists. If you wanted to add a third volume, then what topics would you include?

Stanley: I don't think that a third volume would be appropriate because there are no new chapters that I would want to write. However, there are quite a few topics that I would be interested in adding to existing chapters. These include (1) the kernel method for obtaining generating functions (currently appearing in EC1, second ed., only as Exercise 1.164), (2) Stern's diatomic sequence, (3) further development of lattice paths, of pattern avoidance, of unimodality and log-concavity, and of parking functions, (4) the Laurent phenomenon (in the context of cluster algebras), (5) the theory of left-regular bands and their connection with 
walks on the faces of an arrangement, (6) gen- Mansour: Professor Richard P. Stanley, I eralized permutohedra, (7) combinatorial Hopf would like to thank you for this very interesting algebras, (8) shifted Schur functions, and (9) interview on behalf of the journal Enumerative Schubert polynomials. Sadly, however, I have Combinatorics and Applications.

no plans to write up any of this material. 